Jing Sun* and Nicholas Buys

\title{
Effectiveness of a participative community singing program to improve health behaviors and increase physical activity in Australian Aboriginal and Torres Strait Islander people
}

\begin{abstract}
Background: Aboriginal and Torres Strait Islander individuals continue to experience greater levels of chronic disease than the general Australian population. Evidence suggests that increased sedentary behaviors compared with the traditional healthy and active lifestyles of this population have contributed to this situation. This investigation aimed to examine the health benefits of a community-based singing activity intervention on chronic disease among Aboriginal and Torres Strait Islander Australians.
\end{abstract}

Methods: From 2010 to 2011, 291 Aboriginal and Torres Strait Islander people, from Queensland, Australia, were recruited through five communities to participate in a 1-year community singing program. Participants completed a survey, in the pre- and post-intervention phases of the study, that measured social connectedness, health behavior (e.g., smoking, alcohol consumption), frequency of exercise and art activities, and medication use.

Results: Improvements in health behavior were observed for both male and female participants with chronic disease of all age groups in the community singing program. Improvements in the male and female participants included decreased social loneliness and reduced smoking behavior, increased social support, and increased participation in exercise and art activities.

Conclusions: The participative community singing program was successful in improving the health status and increasing physical activity among Aboriginal and Torres Strait Islander adults.

Keywords: Aboriginal and Torres Strait Islander population; activities; health behaviors; participative community singing.

*Corresponding author: Dr. Jing Sun, School of Public Health and Griffith Health Institute, Griffith University, Gold Coast Campus, Parkland, QLD 4222, Australia, Phone: +61 75552 7875, Fax: +61 75552 8799, E-mail: j.sun@griffith.edu.au
Nicholas Buys: School of Human Services and Social Work and Griffith Health Institute, Griffith University, Gold Coast Campus, Parkland, Gold Coast, QLD, Australia

\section{Introduction}

Aboriginal and Torres Strait Islander Australians continue to experience poorer health status than their nonindigenous counterparts [1-4] in areas such as obesity, chronic and cardiovascular disease (CVD), and cancer [5]. These populations also currently experience poorer health status and poorer quality of life than the general Australian population, including the lowest life expectancy of all Australians [6].

Historically, Aboriginal and Torres Strait Islander Australians had active, healthy lifestyles, relatively free of chronic diseases [7]. The recent increase in chronic conditions in this population has been found to be related to lifestyle factors, including high intake of energy-dense foods, sedentary lifestyles, physical inactivity, smoking and alcohol intake, increased social isolation, and poor social and economic conditions [5]. Currently, an epidemic trend in sedentary behavior exists in Australia, including within the Aboriginal and Torres Strait Islander population [8]. This trend indicates that $15 \%$ of adults Aboriginal and Torres Strait Islander Australians are completely sedentary and $57 \%$ are insufficiently active to maintain good health [8].

Many chronic diseases, such as tobacco smoking and alcohol use, unhealthy diet, lack of physical activity, and low levels of social support share common behavioral and social risk factors [9]. These lifestyle changes, including reduced physical activity, smoking, and increased sedentary behavior and social isolation, are likely contributors to the Aboriginal and Torres Strait Islander Australians peoples' increased rates of obesity, diabetes, and CVD [10, 11]. As a consequence, their life expectancy appears to be similar to that of people in developing countries [12]. 
Most exercise involves activities that are important to prevent obesity, metabolic syndrome, and heart disease, as well as maintain good mental health [13]. However, individual exercise prescriptions generally have had limited success [14]. Instead, community-based exercise programs are generally more successful and experience high participation rates $[15,16]$. A socioecological approach suggests that social support can have an important influence on an individual's overall health and well-being [17]. Social networks and relationships in social settings outside the family provide help in coping and managing stress and can change behaviors in areas such as increased physical activity and promotion of an active lifestyle $[18,19]$. These social networks foster group cohesiveness and interpersonal bonding between members, which encourages commitment and participation in physical activities.

One area of communal activity that has received increasing attention is participative community singing because it entails aerobic exercise, social interaction, and promotion of sense of connectedness [20, 21]. Researchers believe it may be a good avenue in increasing sense of connectedness and promoting participation in exercise activities.

The purpose of this investigation was to evaluate the effectiveness of the Voices United for Harmony community-based singing activity intervention among Australian Aboriginal and Torres Strait Islander populations. This activity was designed to improve health behaviors through increased participation in exercise and to increase social support of individuals wanting to improve their health and activity habits. It was therefore hypothesized that the community singing activity programs, conducted within the local Aboriginal and Torres Strait Islander community, would

- significantly improve participants' health through increases in both aerobic activity and social support;

- lead to increased knowledge about the impact of companionship, support, and encouragement gained through participation in physical activity;

- increase the understanding of its impact on length and frequency of physical activity and overall physical fitness for both sedentary and previously active individuals.

\section{Methods}

From 2010 to 2011, the Voices United for Harmony program was offered to five communities with over 235 Aboriginal individuals in Queensland, Australia. Both the pre- and post-intervention health screenings of potential participants were conducted in these communities. Participating communities included urban, regional, and rural locations throughout the state. Written informed consent was obtained from each participant before pre-intervention screening, and ethical approval was obtained through the Research Ethics Committee at the Griffith University of Australia (GU Ref. No. $\mathrm{PBH} / 13 / 10 /$ HREC).

Our research was community participatory in nature, with local Aboriginal community leaders playing important central roles in the design and implementation of the study. This intervention program was conducted and coordinated, including the recruitment of participants, through local Aboriginal Community Controlled Health Services (CCHSs) representatives. Local CCHSs also assisted with organizing intervention activities, weekly rehearsals, testing, and organization of performances.

Individuals included in the study were those 18 years or older who consented to participate in the intervention program and were able to complete both the assessment and intervention components. Participants included both healthy individuals and those with chronic conditions such as heart disease, diabetes, hypertension, stroke, depression, and schizophrenia. Individuals presenting with medical conditions were referred for medical examination before commencing singing intervention activities.

The present study used a non-randomized study design where participants self-selected a singing intervention activity. The singing programs were of 12 months' duration, from December 2010 to December 2011. Within each community, these programs included weekly group rehearsal sessions for $2 \mathrm{~h}$ per week with 15 -min break for social interaction and encouragement to individually rehearse at home. Each group-singing activity session was organized by a CCHSs Aboriginal community member who had attended leadership training sessions before the program implementation, and the singing activity was led by a professional musician. During singing sessions, participants were able to complete the program within the limits of their physical abilities.

Health surveys were conducted within local Aboriginal communities as part of pre- and post-intervention assessments. Individual characteristics of age, sex, physical activity levels, and personal and/ or family medical history of diabetes, CVD and hypertension, and depression were collected at both these sessions. Singing-related quality of life was measured using the modified Singing Activity Participation Questionnaire [21]. This questionnaire assesses perceived psychological health, physical health, and spiritual health using a 4-point Likert scale, with 0 as "no benefit, as never attended singing" and 4 as "beneficial all the time". Each question is then scored and summed to determine the overall score out of 63 . Individuals who reported completing at least 120 min of singing activity 1 day per week in 3 consecutive months were classified as having achieved the recommended threshold level of singing activity.

Chronic diseases were diagnosed according to standardized protocols established by the Australian standard for heart disease, diabetes, hypertension, stroke, depression, and schizophrenia [22]. Information was also collected regarding the use of medication and previous chronic disease diagnoses.

\section{Statistical analysis}

Statistical analyses were performed using the Statistical Package for Social Science, version 19.0 (SPSS, Chicago, IL, USA). Pre- and postintervention measures were compared using paired t-tests for contin- 
uous variable and $\chi^{2}$ for categorical variables. Pre-training difference between genders were identified using t-tests. Changes in the proportion of participants meeting the physical activity recommendations were evaluated using $\chi^{2}$ analyses. Differences in post-intervention measures between activities were identified using ANCOVA analysis adjusted for age and gender, income, and chronic disease. The association between singing-related health benefits and social connectedness and participation in art and exercise program were analyzed by linear regression analysis adjusted for income and chronic disease conditions. Statistical significance was considered for all statistical analyses where $\mathrm{p}<0.05$.

\section{Results}

Of the 291 Aboriginal individuals who were invited to participate in the Voices United for Harmony Program, 234 consented to participate in the study and attended the pre-intervention assessment. This sample included 115 individuals in the intervention group who participated in pre-intervention assessment sessions and 120 individuals in the control group who participated in the preintervention assessment sessions. There were 76 people in the intervention group and 27 people in the control group in the post-intervention phase. The program attrition rate was $32.7 \%$ for the intervention group and $77.5 \%$ for the control group. However, many participants who were unable to attend post-intervention sessions did complete the intervention programs. Male and female participants were similar in age, ranging from 18 to 75 years (Table 1).

There were no statistically significant differences between the intervention and control groups on the variables age, gender, education, and marital status. There was a statistically significant difference in income between the intervention and control groups, with more people in the control group reporting no income than in the intervention group (see Table 1). Participants attending the pre-intervention assessment sessions were not statistically different from those who were unable to attend in demographic variables such as age, education, and income. Program compliance was fairly high among participants attending the post-intervention assessment, with more than $60 \%$ of males and $75 \%$ of females completing $75 \%-100 \%$ of the intervention activities over the 12-month intervention period. A substantial proportion

Table 1 Participant characteristics.

\begin{tabular}{|c|c|c|c|c|}
\hline & $\begin{array}{r}\text { Intervention } \\
(\mathrm{n}=113), \mathrm{n}(\%)\end{array}$ & $\begin{array}{r}\text { Control } \\
(n=120), n(\%)\end{array}$ & $\chi^{2}$ & p-Value \\
\hline \multicolumn{5}{|l|}{ Age } \\
\hline 18-30 years & $15(13.2)$ & $20(16.8)$ & 1.24 & 0.54 \\
\hline $31-50$ years & $49(43.4)$ & $44(36.7)$ & & \\
\hline 51-78 years & $49(43.4)$ & $56(46.7)$ & & \\
\hline \multicolumn{5}{|l|}{ Gender } \\
\hline Male & $33(29.2)$ & $42(34.7)$ & 0.81 & 0.40 \\
\hline Female & $80(70.8)$ & $79(65.3)$ & & \\
\hline \multicolumn{5}{|l|}{ Marital status } \\
\hline Never married & $33(28.7)$ & 40 (33.9) & 2.69 & 0.61 \\
\hline Widowed & $6(5.2)$ & $9(7.6)$ & & \\
\hline Divorced & $14(12.1)$ & $9(7.6)$ & & \\
\hline Separated & $8(7.0)$ & $10(8.5)$ & & \\
\hline Married & $54(47.0)$ & $50(42.4)$ & & \\
\hline \multicolumn{5}{|c|}{ Highest primary or secondary school education } \\
\hline Did not go to school & $2(1.7)$ & $4(3.4)$ & 5.35 & 0.25 \\
\hline Year 9 or below & $28(24.3)$ & $40(34.2)$ & & \\
\hline Year 10 or equivalent & $27(23.5)$ & $27(23.1)$ & & \\
\hline Year 11 & $21(18.3)$ & $12(10.3)$ & & \\
\hline Year 12 & $37(32.2)$ & $34(29.1)$ & & \\
\hline \multicolumn{5}{|l|}{ Annual income } \\
\hline More than $\$ 20,000$ & $38(41.3)$ & $55(47.0)$ & 8.79 & 0.01 \\
\hline$\$ 1-19,999$ & $36(39.1)$ & $25(21.0)$ & & \\
\hline No income & $18(19.6)$ & $37(31.6)$ & & \\
\hline \multicolumn{5}{|l|}{ Chronic disease } \\
\hline Normal & $15(16.1)$ & $47(47.0)$ & 21.06 & $<0.001$ \\
\hline Chronic conditions & $78(83.9)$ & $53(53.0)$ & & \\
\hline
\end{tabular}

A comparison was made between the intervention and control groups in both the pre- and post-intervention phases. 
of male and female participants presented with chronic diseases (Table 1), and the intervention group had more people with chronic disease then control group.

Both males and females demonstrated a significant increase in participation in physical and other health benefit-related activities over the intervention period $\left(\chi^{2}=21.76, p<0.001\right)$ (see Table 2 ) and significantly improved health behavior. Of particular significance was the proportion of participants in the intervention group who quit smoking $\left(\chi^{2}=21.76, \mathrm{p}<0.001\right)$. The participants in the singing intervention group also demonstrated a significant improvement in individual exercise activities and community exercise, whereas there was a significant decrease in the proportion of participants in individual exercise and community exercise activities in the control group.

The increase in exercise and art activity was significantly greater among males and females in the singing group $(\mathrm{p}<0.001, \mathrm{p}<0.0001)$ in post-intervention phase, compared with the control group. When exercise, social activities, arts, and singing as health benefit measures were combined, the percentage of individuals in the intervention group significantly increased on these measures. For example, $83 \%$ of participants engaged in at least one activity and over $38 \%$ of participants engaged in more than two activities with health benefits. In the control group, $50 \%$ of the participants engaged in exercise activity only in post-intervention phases, which is similar to the percentage of the national population engaged in sedentary behavior [8]. Furthermore, there was a 9.5\% decrease in the percentage of participants in activities with health benefits in the control group.

Changes in health behavior measures by age group were similar over the intervention period. In general, all health behavior measures were similar across age groups. Those who are $\geq 50$ years demonstrated the poorest levels in exercise but the best levels of participation in art-related activities including singing activity. Improvements in activity measures with the singing intervention were observed among singing group participants (Table 2). The singing group participants were different from the control group in the prevalence of chronic diseases in the pre-intervention time. However, after the intervention, improvements in participation in activities with health benefits and health behaviors were observed among this group (Table 2). Medication use decreased among singing group participants (see Table 3), and there were significant improvements in the proportion of participants doing physical activity everyday for $60 \min \left(\chi^{2}=56.72, p<0.0001\right)$ (Table 2).

Following the intervention, similar proportions of male and female participants in the intervention group

Table 2 Participation in health benefit activity and smoking behavior's change.

\begin{tabular}{|c|c|c|c|c|c|c|}
\hline \multirow[t]{2}{*}{ Variables } & \multicolumn{2}{|c|}{ Intervention group } & \multicolumn{2}{|c|}{ Control group } & \multicolumn{2}{|r|}{$\chi^{2}$} \\
\hline & $\begin{array}{r}\text { Pre } \\
(n=115)\end{array}$ & $\begin{array}{r}\text { Post } \\
(n=76)\end{array}$ & $\begin{array}{r}\text { Pre } \\
(n=120)\end{array}$ & $\begin{array}{r}\text { Post } \\
(n=27)\end{array}$ & Pre & Post \\
\hline Exercise, n (\%) & $44(37.3)$ & $23(41.1)$ & $60(49.6)$ & $11(40.0)$ & $3.67^{a}$ & 0.01 \\
\hline Social, n (\%) & $27(22.9)$ & $15(26.8)$ & $12(9.9)$ & $3(10.0)$ & $7.35^{\mathrm{b}}$ & 2.39 \\
\hline Arts, n (\%) & $34(34.3)$ & $36(69.2)$ & $13(11.8)$ & $1(5.6)$ & $15.17^{c}$ & $21.76^{c}$ \\
\hline Combined, n (\%) & $61(61.6)$ & $43(82.7)$ & $65(59.1)$ & $14(50.0)$ & 0.14 & $11.04^{b}$ \\
\hline Smoking, n (\%) & $38(32.5)$ & $11(20.0)$ & $51(42.9)$ & $16(60.0)$ & 2.71 & $11.04^{c}$ \\
\hline
\end{tabular}

A comparison was made between the intervention and control groups in both the pre- and post-intervention phases. For $\chi^{2}$, Pre is the comparison between the intervention and control groups in the pre-intervention phase, whereas Post is the comparison between the intervention and control groups in the post-intervention phase.

${ }^{\mathrm{a}} \mathrm{p}<0.05,{ }^{\mathrm{b}} \mathrm{p}<0.01,{ }^{\mathrm{c}} \mathrm{p}<0.001$.

Table 3 Medication use.

\begin{tabular}{|c|c|c|c|c|c|c|}
\hline \multirow[t]{2}{*}{ Variables } & \multicolumn{2}{|c|}{ Intervention group } & \multicolumn{2}{|r|}{ Control group } & \multicolumn{2}{|r|}{$\chi^{2}$} \\
\hline & Pre $(n=115)$ & Post $(n=76)$ & Pre $(n=120)$ & Post $(n=27)$ & Pre & Post \\
\hline Medication use, n (\%) & $59(51.3)$ & $48(63.0)$ & $77(64.5)$ & $21(78.9)$ & $4.24^{\mathrm{a}}$ & 1.63 \\
\hline Changed medication, n (\%) & $25(22.0)$ & $21(27.8)$ & $15(12.5)$ & $7(26.3)$ & 2.40 & 1.56 \\
\hline Duration of medication use, days & $934(1866)$ & $1828(2830)$ & $125(113)$ & $2543(2776)$ & $\mathrm{t}=3.82^{\mathrm{b}}$ & $\mathrm{t}=-0.88$ \\
\hline
\end{tabular}

${ }^{\mathrm{a}} \mathrm{p}<0.05,{ }^{\mathrm{b}} \mathrm{p}<0.01$. 
reported physical activity levels meeting national physical activity recommendations in the post-intervention phase when compared with the pre-intervention phase $\left(\chi^{2}=13.23, p<0.001\right)$. The proportions of sufficiently active individuals increased among singing program participants $\left(\chi^{2}=36.25, p=0.0001 ; \chi^{2}=14.71, p=0.0001\right)$. Similar increases in proportions of participants meeting physical activity recommendations were observed for all age groups in the singing intervention group (18-29 years: $\chi^{2}=12.06, \mathrm{p}=0.0005 ; 30-49$ years: $\chi^{2}=19.38, \mathrm{p}<0.0001$; 50 years and older: $\left.\chi^{2}=15.25, \mathrm{p}<0.0001\right)$. Furthermore, a higher proportion of singing intervention group participants significantly reduced their smoking behaviors compared with control group participants ( $20 \%$ vs. $80 \%$, $\chi^{2}=11.04 ; \mathrm{p}<0.001$.

The interaction between groups (intervention vs. control) and study time (pre- and post-intervention) on social connectedness was analyzed using univariate analysis of variance. The results indicate that there was a significant interaction between group and time $(\mathrm{F}=6.23, \mathrm{p}<0.01$; see Figure 1). This suggests that there was a significant increase in the sense of connectedness in the intervention group and a significant decrease in the sense of social connectedness in the control group over the 12-month intervention period and that this difference increased over time. The benefits of singing are significantly related to social connectedness (see Table 4), which may act as a significant factor to promote physical exercise and arts activities. The effect of social connectedness on the increase of participation in exercise and art activities was further analyzed by logistic regression analysis (see Table 5). The results indicate that social connectedness has a significant effect on exercise and art activity participation in both
Table 4 Relationship between community singing and sense of connectedness.

\begin{tabular}{lr}
\hline & Sense of connectedness, B (interval) \\
\hline Community singing & $0.44(0.21 \text { to } 0.68)^{\text {a }}$ \\
Age & $-0.48(-1.83$ to 0.87$)$ \\
Gender & $-2.59(-6.55$ to 1.36$)$ \\
Income & $0.05(-2.03$ to 2.13$)$ \\
Variance explained, \% & 7 \\
\hline
\end{tabular}

${ }^{a} p<0.05$. Linear regression analysis was used to analyze the association between participation in community singing and sense of social connectedness.

the intervention and control groups. The effect is significant even when age, gender, and income as confounding factors were controlled in the analysis.

\section{Discussion}

This study is the first of its type to investigate the effectiveness of a community-based singing activity program for Aboriginal and Torres Strait Islander Australian individuals on health. Improvements in health behavior and physical activity were observed among participants of both genders and across age groups in the intervention group. This is especially notable given that the singing intervention group participants also demonstrated poorer health status and a high prevalence of sedentary behaviors in the pre-intervention phase. As individuals with the greatest health risk experienced the greatest improvements with intervention, these results indicate that

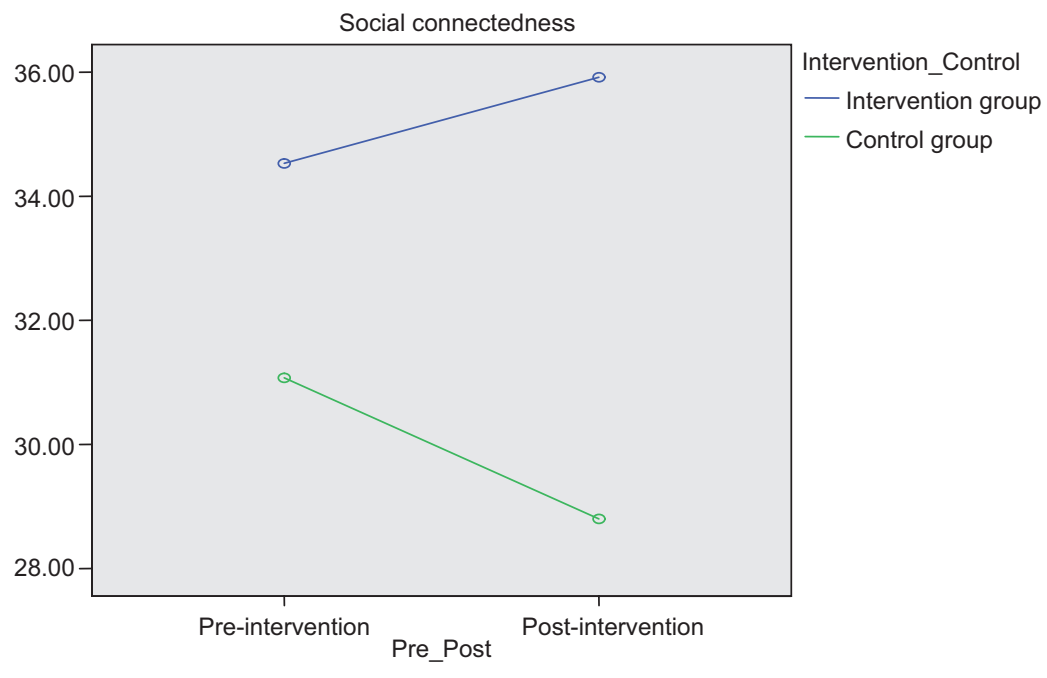

Figure 1 Interaction effect on group (intervention and control group) and intervention time (pre- and post-intervention time). 
Table 5 Relationship between sense of connectedness and participation of exercise and art activities.

\begin{tabular}{|c|c|c|c|}
\hline & Individual exercise & Community exercise & Arts \\
\hline Social connectedness & 0.99 (0.97 to 1.02$)$ & $1.05(1.00 \text { to } 1.10)^{\mathrm{a}}$ & $1.03(1.00 \text { to } 1.07)^{\mathrm{a}}$ \\
\hline \multicolumn{4}{|l|}{ Age } \\
\hline 18-29 years & $0.39(0.16 \text { to } 0.61)^{b}$ & $-0.01(-0.26$ to 0.28$)$ & $-0.14(-0.36$ to 0.09$)$ \\
\hline $30-49$ years & $0.18(-0.01$ to 0.37$)$ & $0.07(-0.15$ to 0.28$)$ & $-0.16(-0.35$ to 0.04$)$ \\
\hline$\geq 50$ years & $0.04(-0.15$ to 0.23$)$ & $-0.03(-0.24$ to 1.80$)$ & $-0.02(-0.21$ to 0.17$)$ \\
\hline Gender (male) & $0.49(0.23$ to 1.05$)$ & $1.81(0.61$ to 5.33$)$ & $1.90(0.76$ to 4.75$)$ \\
\hline \multicolumn{4}{|l|}{ Income } \\
\hline$>\$ 20,000$ (reference) & 1 & 1 & 1 \\
\hline$\$ 1-19999$ & $1.58(0.65$ to 3.86$)$ & $1.06(0.32$ to 3.53$)$ & $0.75(0.26$ to 2.13$)$ \\
\hline No income & $0.68(0.31$ to 1.52$)$ & $1.45(0.52$ to 4.05$)$ & 1.40 (0.58 to 3.39$)$ \\
\hline Variance explained, \% & 20.5 & 9.7 & 11.2 \\
\hline
\end{tabular}

Dependent variables are exercise, social exercise, and arts. Independent variables are social connectedness, age, gender, and income. ${ }^{a} p<0.05,{ }^{b} p<0.001$. Linear regression analysis was used to analyze the association between social connectedness and participation in exercise and art activities.

Aboriginal and Torres Strait Islander Australian adults were able to identify a need to participate in aerobic activity programs appropriate to their level of fitness, physical ability, and health status. Although the singing program is an aerobic activity and less physically intense than other sport programs, participants with poorer health status selected this program and achieved a greater reduction in chronic disease-related risk factors. Activity scores in relation to physical activity, arts, and social participation and increased proportions of participants are meeting national physical activity recommendations among participants of the singing program.

The improvements in health behavior and participation level in exercise indicate that a community singing program of this type is appropriate for individuals of varying age and health status. Increases in participation in exercise activity levels were observed among most participants in the singing groups, and individuals with the greatest health risk experienced the greatest improvements with the community singing program. This is consistent with the findings of Hampshire and Matthijsse [23] that the youths in the singing group were more likely to do other extracurricular activities than those in the control group. The possible reason is that the youths in the singing group have more opportunities to establish friendship, and this is important to those who struggle socially. Friendship was also a prominent theme in Aboriginal and Torres Strait peoples' accounts of group singing. Many participants made new friends though Voices United for Harmony Project. The intervention participants felt more socially connected than those in the control group, and this may have significantly contributed to their improvement in health behavior and increased their participation in the extra exercise activities.
The significant increase in the sense of social connectedness in the intervention group and the concomitant increase in participation in exercise and art activities support a socioecological approach, which suggests that social connectedness has an important influence on an individual's overall health and well-being. Social networks and relationships in social settings help in increasing participation in physical activity and in achieving an active lifestyle.

Few published culturally appropriate activity interventions have been conducted with Aboriginal and Torres Strait Islander populations, with most having small sample sizes and lacking group-singing interventions. Other activity interventions among Aboriginal and Torres Strait Islander populations have met with variable success. Community-based group exercise programs have led to improvements in relation to reduced risk factors in chronic disease conditions [19]. Similar to those results, our current community singing program not only promoted more participation in a singing program but also increased the proportion of participants in extra exercise, aerobic, and other types of art activities. These results suggest that a community-based intervention is likely to be more successful among Aboriginal and Torres Strait Islander populations. Given the high rates of obesity, diabetes, CVD, and other chronic conditions among this population, community-based singing activity interventions should be implemented among the Australian Aboriginal and Torres Strait Islander populations to combat their poor health status. The success of this intervention among all adults within the communities highlights this type of intervention as an appropriate health improvement strategy for reducing and preventing the chronic disease experience among this population. Implementing 
community-based singing programs within local communities provides an avenue for improving and maintaining the health of all Australian Aboriginal and Torres Strait Islander adults.

The evaluation of this intervention program is limited by the "self-selection" nature of the study design, and there is a high attrition rate in the control group. To further investigate the effectiveness of community-based singing programs in Australian Aboriginal communities, future investigations should randomize participants to intervention programs for effective comparison and an incentive program should be provided to control group participants to engage them in the project. Another limitation is that the study was only conducted in Queensland, so we are unsure of its generalizability to other states or populations. It is recommended that a national study be conducted to determine the wider applicability of these findings.

\section{Conclusions}

Our study suggests that a community-based singing intervention is likely to be more successful among Aboriginal and Torres Strait Islander Australian populations than individual-based singing programs. Given the high rates of obesity, diabetes, CVD, and other chronic conditions among this population, it is therefore recommended that these types of interventions should be implemented widely among the Australian Aboriginal populations to combat their poor health status. Implementing communitybased singing activity programs within local Aboriginal communities provides an avenue for reducing high-risk factors in chronic diseases and maintaining the health of all Aboriginal and Torres Strait Islander Australian adults, thereby addressing a significant area of health disadvantage for this population.

Acknowledgements: The authors received financial support from Griffith Health Institute and the Provost Chancellor Office at Logan Campus at Griffith University, Queensland Centre for Social Innovation, and Queensland Aboriginal and Islander Health Council. The authors also wish to acknowledge the support of the following Aboriginal Community Controlled Health Services: Kambu Medical Service Centre Pty Ltd., Kalwun Health Service, Aboriginal and Torres Strait Islander Community Health Service Brisbane Ltd., Goolburri Health Advancement Corporation, and Warwick Community. The authors also wish to thank all the participants from the five Aboriginal and Torres Strait Islander communities.

\section{Conflict of interest statement}

Authors' conflict of interest disclosure: The authors stated that there are no conflicts of interest regarding the publication of this article. Financial support played no role in the study design; in the collection, analysis, and interpretation of data; in the writing of the report; or in the decision to submit the report for publication.

Research funding: None declared. Employment or leadership: None declared. Honorarium: None declared.

Received October 22, 2012; accepted October 25, 2012

\section{References}

1. Australian Bureau of Statistics. National health survey: summary of results Australia 2004-05. Canberra, Australia: Australian Bureau of Statistics, 2006.

2. Australian Bureau of Statistics. National survey of mental health and wellbeing: summary of results. Contract No. 4326.0. Canberra, Australia: Australian Bureau of Statistics, 2007.

3. Brough M, Bond C, Hunt J, Jenkins D, Shannon C, Schubert L. Social capital meets identity: aboriginality in an urban setting. J Soc 2006;42:396-411.

4. Butler T, Allnutt S, Kariminia A, Cain D. Mental health status of Aboriginal and non-Aboriginal prisoners. Aust NZ J Psychiatry 2007;41:429-35.

5. Australian Institute of Health and Welfare. Measuring the social and emotional wellbeing of Aboriginal and Torres Strait Islander peoples. Report No.: In Cat. No. IHW24. Canberra, Australia: AlHW, 2009:115.
6. Australian Institute of Health and Welfare and Australian Bureau of Statistics. The health and welfare of australia's Aboriginal and Torres Strait Islander peoples. Report No.: ABS Cat. No. 4704.0. Canberra, Australia: AlHW, 2008.

7. Australian Aboriginal and Torres Strait Islander Social Justice Commissioner. Social justice report. Report No. 3/2005. Sydney, NSW, Australia: Australian Aboriginal and Torres Strait Islander Social Justice Commissioner, 2005.

8. Bauman A, Sallis JF, Dzewaltowski DA, Owen N. Toward a better understanding of the influences on physical activity: the role of determinants, correlates, causal variables, mediators, moderators, and confounders. Am J Prev Med 2002;23:5-14.

9. World Health Organization. World health report 2002. Geneva: WHO, 2003.

10. Australian Institute of Health and Welfare. Australia's health. Report No.: Cat. No. AUS 44. Canberra, Australia: AlHW, 2004. 
11. Australian Bureau of Statistics. Australian social trends 2007: selected chronic conditions among Aboriginal and Torres Strait Islander peoples. Report No.: NO. 4102.0. Canberra, Australia: Australian Bureau of Statistics, 2007.

12. Australian Bureau of Statistics. National Aboriginal and Torres Strait Islander social survey (2004). Contract No.: ABS Cat. No. 4714.0. Canberra, Australia: Australian Bureau of Statistics, 2004.

13. Foulds HJ, Bredin SS, Warburton DE. The effectiveness of community based physical activity interventions with Aboriginal peoples. Prev Med 2011;53:411-6.

14. Wing RR, Tate DF, Gorin AA, Raynor HA, Favam JL. A self-regulation program for maintenance of weight loss. $\mathrm{N}$ Engl J Med Overseas Ed 2006;355:1563-71.

15. Cohen GD, Perlstein S, Chapline J, Kelly J, Firth KM, Simmens S. The impact of professionally conducted cultural programs on the physical health, mental health, and social functioning of older adults. Gerontologist 2006;46:726-34.

16. Cohen GD, Perlstein S, Chapline J, Kelly J, Firth KM, Simmens S. The impact of professionally conducted culturally programs on the physical health, mental health and social functioning of older people -2 year results. J Aging Hum Arts 2007;1:5-22.

17. First MB, Gibbon M, Spitzer RL, Williams JB. Structured clinical interview for DSM-IV axis I disorders: nonpatient edition
(SCID-I/NP). New York: Biometrics Research Department, New York State Psychiatric Institute, 1996.

18. Springer AE, Kelder SH, Hoelscher DM. Social support, physical activity and sedentary behavior among 6th-grade girls: a crosssectional study. Int J Behav Nutr Phys Act 2006;3:1-10.

19. Kim X, McEwen LN, Kieffer EC, Herman WH, Piette JD. Self-efficacy, social support, and associations with physical activity and body mass index among women with histories of gestational diabetes mellitus. Diabetes Educ 2008;34: 719-28.

20. Clift S, Hancox G, Morrison I, Hess B, Kreutz G, Stewart D. Choral singing and psychological wellbeing: quantitative and qualitative findings from English choirs in a cross-national survey. J Appl Arts Health 2010;1:19-34.

21. Clift S, Morrison I. Group singing fosters mental health and wellbeing: findings from the East Kent "singing for health" network project. Mental Health Soc Inclusion 2011;15: 88-97.

22. National Heart Foundation of Australia. Quick guide to management of hypertension. National Heart Foundation of Australia: Canberra, 2010.

23. Hampshire KR, Matthijsse M. Can arts projects improve young people's wellbeing? A social capital approach. Soc Sci Med 2010;71:708-16. 